\title{
Association of adrenal insufficiency with insulin-dependent diabetes mellitus in a patient with inactivating mutations in nicotinamide nucleotide transhydrogenase: a phenocopy of the animal model
}

\author{
Rachel Scott, Guy Van Vliet and Johnny Deladoëy \\ Endocrinology Service and Research Center of the Sainte-Justine Hospital and Department of Pediatrics, \\ University of Montreal, Montreal, Quebec, Canada
}

We read with great interest the paper of RoucherBoulez et al. in the July issue of the Journal reporting extra-adrenal phenotypes (i.e. gonadal, cardiac and thyroidal disorders) in a cohort of 18 patients with adrenal insufficiency due to nicotinamide nucleotide transhydrogenase (NNT) mutations (1). Indeed, NNT loss of function, which hampers NADPH production in mitochondria, was initially reported in families with familial isolated glucocorticoid deficiency (2). Yet, Nnt expression is severely reduced in pancreas of C57BL/6J mice due to a naturally occurring loss-of-function Nnt mutation in this strain. Consequently, C57BL/6J mice have reduced insulin secretion because of increased oxidative stress in pancreatic beta-cells (3). Here, we report a patient with NNT mutations who developed diabetes mellitus, thus recapitulating the Nnt-deficient C57BL/6J mouse phenotype (3).

A 12-month-old previously healthy boy was admitted with severe retro-pharyngeal cellulitis, causing airway obstruction, thrombosis of the left jugular vein, compression of the left carotid artery and associated infarction of the left parietal lobe. After treatment of the infection, anorexia and vomiting persisted, with a loss of $18 \%$ of body weight over a period of 6 weeks despite tube feeding. Serum sodium was low $(126 \mathrm{mmol} / \mathrm{L})$ and potassium was high $(8.7 \mathrm{mmol} / \mathrm{L})$. Serum cortisol was $<22 \mathrm{nmol} / \mathrm{L}$ and ACTH (466 pmol/L) and renin (202 ng/L) were very high, confirming primary adrenal insufficiency. The patient responded rapidly to glucocorticoid and mineralocorticoid replacement. We previously reported the patient as broadening the phenotype of NNT mutants to combined glucocorticoid and mineralocorticoid deficiencies (4). At 9.5 years, the patient presented with a two-week history of polyuria, polydipsia and weight loss. A glucose level of $42 \mathrm{mmol} / \mathrm{L}$ confirmed diabetes mellitus. There was no acidosis. Anti-GAD antibodies were slightly positive at 3.6 (normal <1), but anti-IA2 antibodies were negative. The patient is now 12 years old and requires 1 unit/kg/day of insulin to maintain good metabolic control.

The NNT mutations in this patient have already been described (patient \#12 in Ref. (2)), as have the two heterozygous (biallelic) variants in the gene $M E 3$ revealed by exome sequencing, which may explain the combined glucocorticoid and mineralocorticoid deficiencies (4). The purpose of the present report is to add insulin-dependent diabetes mellitus to the phenotypic spectrum of patients carrying NNT mutations, consistent with findings in the animal model (3).

Declaration of interest

The authors declare that there is no conflict of interest that could be perceived as prejudicing the impartiality of this commentary.

Funding

J D and G V V are supported by the Girafonds/Fondation du CHU SainteJustine.

Published by Bioscientifica Ltd. 


\section{References}

1 Roucher-Boulez F, Mallet-Motak D, Samara-Boustani D, Jilani H, Ladjouze A, Souchon PF, Simon D, Nivot S, Heinrichs C, Ronze $\mathrm{M}$ et al. 2016 NNT mutations: a cause of primary adrenal insufficiency, oxidative stress and extra-adrenal defects. European Journal of Endocrinology 175 73-84. (doi:10.1530/EJE-16-0056)

2 Meimaridou E, Kowalczyk J, Guasti L, Hughes CR, Wagner F, Frommolt P, Nurnberg P, Mann NP, Banerjee R, Saka HN et al. 2012 Mutations in NNT encoding nicotinamide nucleotide transhydrogenase cause familial glucocorticoid deficiency. Nature Genetics 44 740-742. (doi:10.1038/ng.2299)

3 Toye AA, Lippiat JD, Proks P, Shimomura K, Bentley L, Hugill A, Mijat V, Goldsworthy M, Moir L, Haynes A et al. 2005 A genetic and physiological study of impaired glucose homeostasis control in C57BL/6J mice. Diabetologia 48 675-686. (doi:10.1007/s00125-0051680-z)

4 Hasselmann C, Deladoey J, Vuissoz JM, Patry L, Alirezaie N, Schwartzentruber J, Deal C, Van Vliet G, Majewski J \& Samuels M 2013 Expanding the phenotypic spectrum of nicotinamide nucleotide transhydrogenase (NNT) mutations and using whole exome sequencing to discover potential disease modifiers. Journal of Genomes and Exomes 1 19-30. (doi:10.4137/JGE. S11378)

Received 24 November 2016

Accepted 5 December 2016 